\title{
CT Hepatic Venography: 3D Vascular Segmentation for Preoperative Evaluation
}

\author{
Catalin Fetita $^{1}$, Olivier Lucidarme ${ }^{2}$, Françoise Prêteux ${ }^{1}$, and Philippe Grenier ${ }^{2}$ \\ 1 ARTEMIS Project Unit, INT, Groupe des Ecoles des Télécommunications, \\ 9 rue Charles Fourier, 91011 Evry Cedex, France \\ 2 Central Radiology Service, Pitié Salpêtrière Hospital, \\ 47-83 boulevard de l'Hôpital, 75651 Paris Cedex 13
}

\begin{abstract}
Preventing complications during hepatic surgery in livingdonor transplantation or in oncologic resections requires a careful preoperative analysis of the hepatic venous anatomy. Such an analysis relies on CT hepatic venography data, which enhances the vascular structure due to contrast medium injection. However, a 3D investigation of the enhanced vascular anatomy based on typical computer vision tools is ineffective because of the large amount of occlusive opacities to be removed. This paper proposes an automated 3D approach for the segmentation of the vascular structure in CT hepatic venography, providing the appropriate tools for such an investigation. The developed methodology relies on advanced topological and morphological operators applied in monoand multiresolution filtering schemes. It allows to discriminate the opacified vessels from the bone structures and liver parenchyma regardless of noise presence or inter-patient variability in contrast medium dispersion. The proposed approach was demonstrated at different phases of hepatic perfusion and is currently under extensive validation in clinical routine.
\end{abstract}

\section{Introduction}

Liver surgery raises several challenges in terms of preoperative evaluation, both in the case of living-donor liver transplantation and oncologic resections. The key issue is to take into account the intrahepatic vessel anatomy specific to each patient in order to reduce surgical complications and/or planning a resection strategy 12. The hepatic vascular system is composed of three hierarchical networks: portal tree, hepatic veins and hepatic arteries. Among them, the portal system defines the functional units of the liver while the hepatic veins are in charge with liver drainage. Their morphological analysis is thus mandatory at the preoperative stage in order to define virtual anatomical landmarks, a safety margin for resection, and the zone impacted by a vessel ligation. CT hepatic venography provides a vessel investigation modality based on MDCT data acquisition after injection of a contrast agent. The hepatic vasculature is progressively opacified and the different vascular systems irrigating the liver become visible. Developing computer vision tools for preoperative planning based on such data is still an active research area despite major advances in this field. Several virtual/augmented reality systems have been proposed for liver surgery planning, 


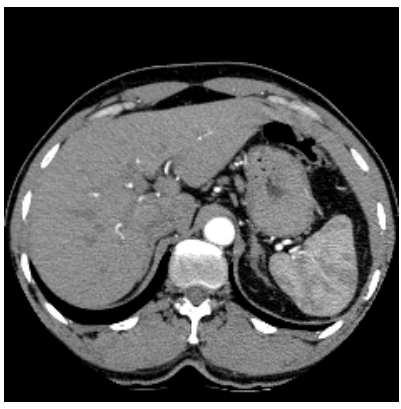

(a) Arterial phase.

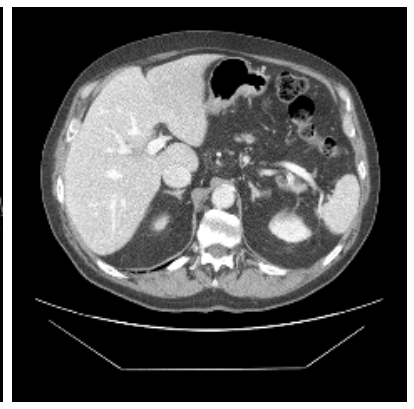

(b) Portal phase.

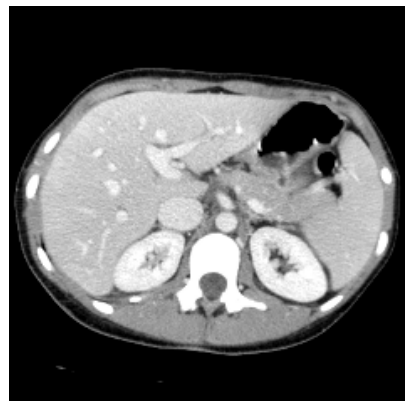

(c) Venous phase.

Fig. 1. Some examples of CT hepatic venography axial images showing a strong noise, different levels of liver opacifying and vertebra-aorta artifact connections

relying on complex interaction interfaces $3,4 / 5,6]$ and different visualization and segmentation strategies 1 1/7819. Note that, solving the liver segmentation problems, generally requires excellent radiological data which is not always achievable in clinical routine. Strong noise, different levels of liver opacifying and partial volume effects resulting in bones-vessels artifact connections on CT images are the major difficulties to overcome (Fig. 1).

This paper addresses the issue of the automatic 3D segmentation of hepatic vessels in clinical CT hepatic venography and develops an original approach based on advanced topological and morphological operators combined in monoand multiresolution filtering schemes. The proposed approach was applied to CT data acquired at different phases of liver perfusion (arterial, portal and venous, Fig. (1) in order to test its robustness with respect to anatomical variability.

The paper is organized as follows. Section 2 presents the multiresolution framework on which the segmentation approach relies, and introduces the 3D morphological operator involved. Section 3 describes and illustrates the developed segmentation strategy. Results are presented and discussed in Section 4.

\section{A Multiresolution Segmentation Framework}

In order to overcome the limitations related to the strong noise and to the variable level of liver opacifying, a multiresolution approach is used for vascular segmentation. Such an approach relies on a morphological operator, so-called selective marking and depth constrained connection cost (SMDC-CC).

Defined on functions $f: X \subset \Re^{n} \rightarrow \Re$ of connected support $\operatorname{supp}(f)=X$ and upper bounded, on any bounded subset of $\operatorname{supp}(f)$, the SMDC-CC affects the local minima of $f$ according to connectivity and morphometric criteria. While a complete mathematical definition of SMDC-CC can be found in [10, its intuitive interpretation will be given in the following. Let us imagine $f$ as the surface of a relief. A point $x \in \operatorname{supp}(f)$ is called topographically connected to a subset $Y \subset \operatorname{supp}(f)$, if there is a descending path on $f$ leading from $x$ to $Y$. Computing 


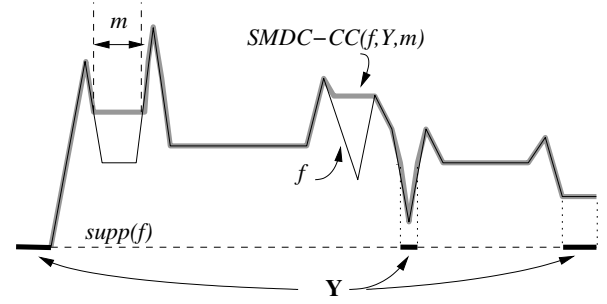

(a) 1-D example.
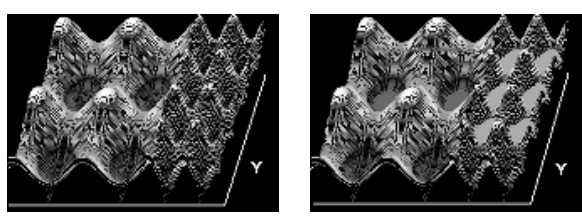

(b) 2-D example ( $f$ left, $S M D C-C C$ right).

Fig. 2. SMDC-CC of $f$ with respect to a $Y$ subset, for a given SE size, $m$

SMDC-CC of $f$ with respect to a non-empty subset $Y \subset \operatorname{supp}(f)$ will result in "filling in", at constant level, all local minima of $f$ topographically disconnected from $Y$. The "filling in" level is controlled by the size $m$ of the structuring element (SE) associated with the SMDC-CC operator. If such a SE is a n-D ball, the "filling in" depth of a "valley" is given by the level at which the "valley" width becomes larger than $m$ (Fig. 2).

By adjusting the $m$ parameter, the SMDC-CC can be implemented in a multiresolution scheme, making it possible to segment structures of interest in a noisy environment. Such a scheme is visually described in Fig. 3. simulating the detection of the vascular structure in a liver-like, noisy environment (Fig. 3(a)) The reference $Y$ subset is defined as the image border. The SMDC-CC is first applied on the negative data (Fig. 3(b) with a small-size $m$ to filter out the local minima corresponding to the noise (Fig. 3(c) $)$. The small-size vasculature is not affected at this step due to the topographically connection with large caliber vessels. In a second step, by increasing the $m$ size over the maximum vessel caliber, the SMDC-CC selects the desired structures (Fig. 3(d)] . A histogrambased thresholding of the SMDC-CC difference (Fig. 3(e) provides the vessel segmentation (Fig. 3(f)].

Note that the local maxima in the original data (white areas) which are situated at boundary and connected to $Y$ (image border) were not segmented. This property will be useful for designing an additional vessels-bones discrimination approach in CT hepatic venography data, exploiting the described multiresolution framework.

\section{An Automated Approach for CT Venography Segmentation}

Looking back at the CT images from Fig. 1, we note that the real hepatic venography data raise additional challenges for vessel segmentation. Such challenges are related to the automated discrimination between the opacified vessels and the bone structures. Since density or morphological criteria are neither accurate nor robust for this purpose, the idea is to filter the original data in order to achieve the appropriate configuration for a SMDC-CC based segmentation 


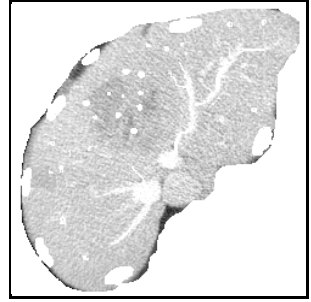

(a)

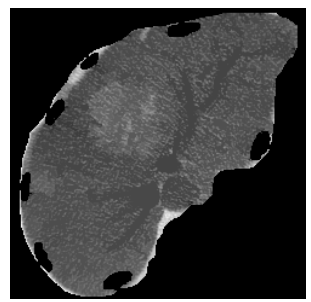

(d)

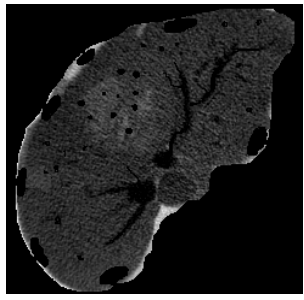

(b)

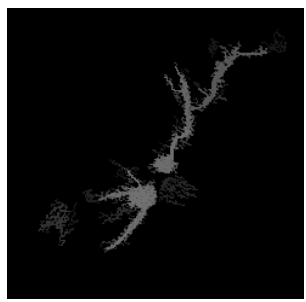

(e)

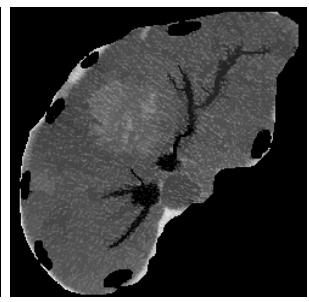

(c)

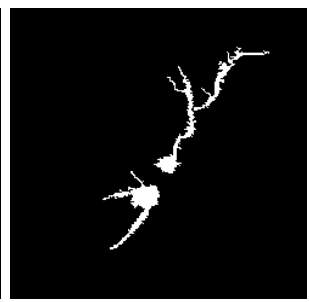

(f)

Fig. 3. SMDC-CC based multiresolution segmentation scheme: (a) original data, (b) negative data, (c) noise filtering with SMDC-CC of small-size $m$, (d) vessel selection with SMDC-CC of large-size $m$, leaving unaffected the "valleys" connected with the $Y$ subset (image border), (e) difference (d)-(c), (f) adaptive thresholding of (e)

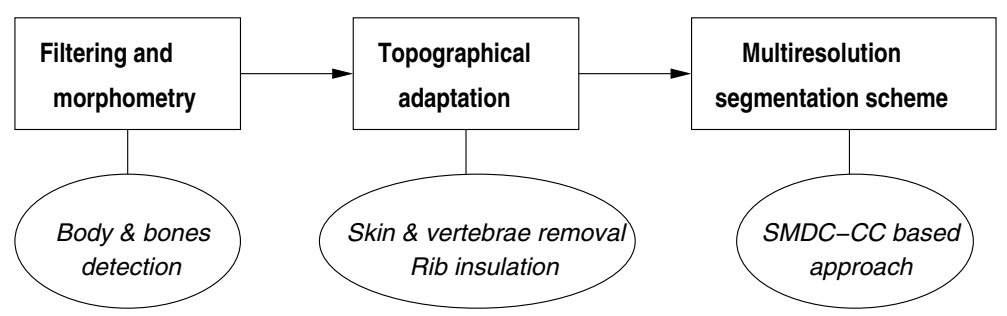

Fig. 4. Block diagram of the implemented segmentation approach

(Fig. 3(a) . More precisely, after the filtering step, the bones have to become topographically connected to the $Y$ subset and disconnected from the opacified vessels. The latter condition need to be checked because of the presence of noise which, combined with partial volume effects, may produce on CT images an artifact connectivity between vertebrae and aorta (Fig. 1(b)].

Based on the previous considerations, the developed segmentation scheme consists of three modules, as illustrated in the block diagram from Fig. 4. The first module performs a morphological filtering for detecting the body and the bones, and extracting the parameters involved in the multiresolution segmentation scheme. The second module provides the topographical adaptation imposed by the multiresolution framework, by removing the skin and the vertebrae and insulating the ribs. The third module achieves the segmentation as discussed 


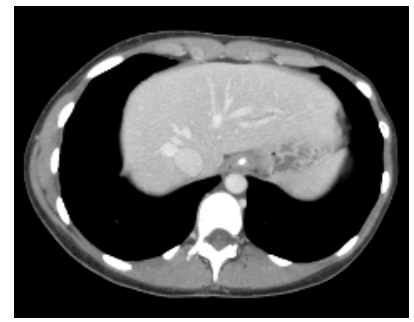

(a) Original axial image, $I_{o}$.

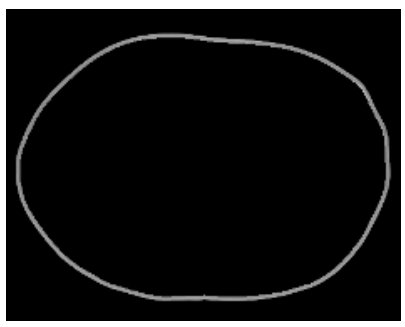

(d) $I_{\text {skin }}$.

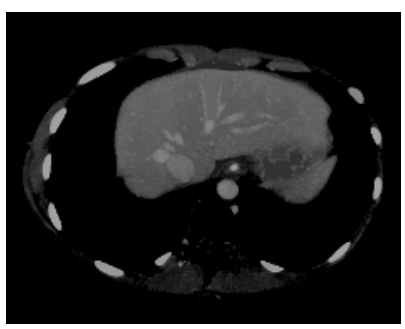

(g) $I_{n v}$.

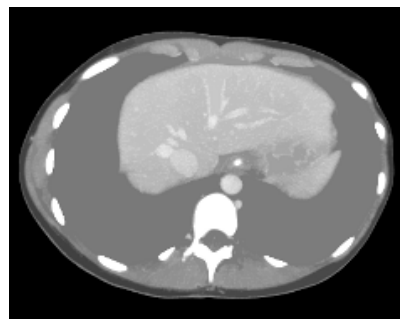

(b) $I_{f}=C C\left(I_{o}\right)$.

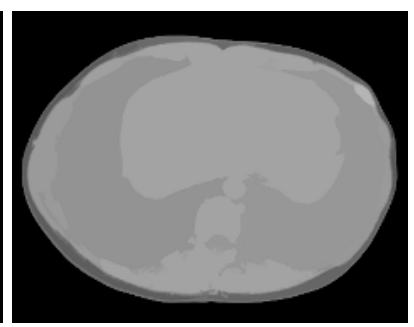

(e) $I_{r g}=G R\left(I_{f}, I_{s k i n}\right)$.

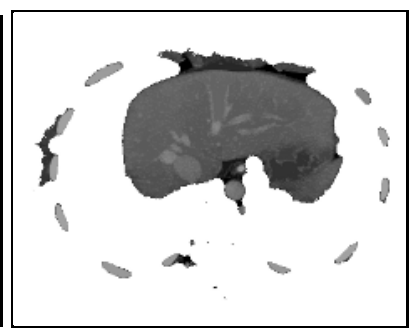

(h) $I_{\text {topo }}$.

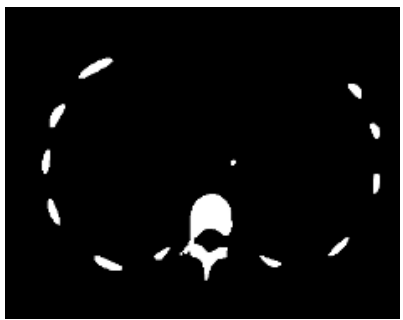

(c) $I_{\text {bone }}=F H\left(\operatorname{Bin}_{255}^{255} I_{f}\right)$.

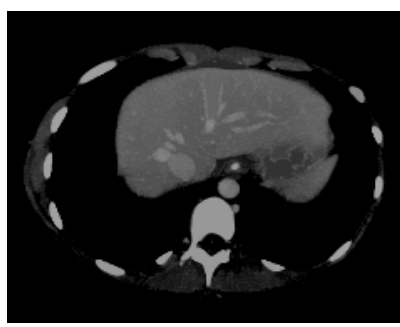

(f) $I_{l i v}=I_{f}-I_{r g}$.

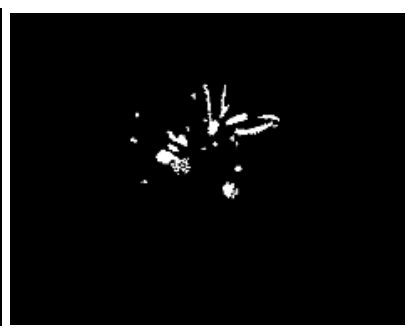

(i) $I_{\text {seg }}=M R\left(I_{\text {topo }}\right)$.

Fig. 5. Stepwise illustration of the 3D segmentation algorithm presented in the Fig. 4 block diagram. The following notations were used: $C C$ - connection cost with respect to borders, $\operatorname{Bin}_{a}^{b}$ - binarization between the thresholds $a$ and $b, F H($.$) - "fill holes"$ operator, $G R\left(I_{1}, I_{2}\right)$ - gray-level reconstruction of $I_{1}$ by $I_{2}$, and $M R$ - multiresolution segmentation approach $c f$.

in 92 . The different steps of the segmentation approach are described in the following and illustrated in Fig. 5 at the level of an axial CT image (Fig. 5(a)].

\section{A. Filtering and morphometry}

1. The CT data is filtered by means of a connection cost (CC) operator 12 applied with respect to the volume borders $\left(I_{f}\right.$, Fig. 5(b) . Such an operator performs like a SMDC-CC one, with $m=\infty$. It regularizes the noise, levels up the local minima and provides the threshold value for body detection.

2. The bone components and the highly opacified vessels are extracted by binarization followed by a $2 \mathrm{D}$ "fill holes" procedure $\left(I_{\text {bone }}\right.$, Fig. $5(\mathrm{c})$. The max- 
imum axial size of the vertebrae, further denoted by $V S$, is computed along the volume data using a $2 \mathrm{D}$ distance map on $I_{\text {bone }}$ with respect to the background.

\section{B. Topographical adaptation}

3. Based on the distance map computed with respect to the bone structures outside the body volume, an estimated of the minimal thickness of the skin and muscle tissues outside the rib cage is computed $\left(I_{\text {skin }}\right.$, Fig. 5(d)). The body "background" $\left(I_{r g}\right.$, Fig. $5(\mathrm{e})$ is computed by means of a 3D gray-level reconstruction [1] of $I_{f}$ by $I_{s k i n}$. The soft tissue outside the liver is then removed $\left(I_{l i v}\right.$, Fig. $\left.5(\mathrm{f})\right)$ by subtracting from the body volume, $I_{f}$, its "background", $I_{r g}$.

4. The spine is detected from the bone data, $I_{b o n e}$, and removed from $I_{l i v}$ in order to prevent any artifact connection with aorta $\left(I_{n v}\right.$, Fig. $\left.5(\mathrm{~g})\right]$. The procedure is based on 3D morphological opening of size $V S / 2$ (removing the ribs and the other dense structures from $I_{b o n e}$ ) followed by a geodesic dilation with a small-size spherical structuring element (SE).

5 . In order to achieve the configuration requested in 92 , the ribs and the liver are insulated with maximum gray-level regions $\left(I_{\text {topo }}\right.$, Fig. 5(h) by means of $3 \mathrm{D}$ region growing with a spherical SE of radius $V S / 2$, initiated at the volume lateral walls.

\section{Multiresolution segmentation scheme}

6. The multiresolution framework based on the SMDC-CC operator is applied to $I_{\text {topo }} c f$. $\$ 2$ in order to achieve the vessel segmentation $\left(I_{\text {seg }}\right.$, Fig. $\left.5(\mathrm{i})\right)$. The reference subset $Y$ is defined as the volume border, while the SE size is respectively set to $m=2$, for noise removal, and to $m=V S$, for vascular selection.

\section{Results and Discussion}

The robustness of the developed vessel segmentation approach was tested on a CT hepatic venography database acquired in clinical routine in 10 patients at different phases of liver perfusion. No constraint was imposed on the acquisition protocol. MDCT data were provided by the Central Radiology Service of Pitié Salpêtrière Hospital in Paris which used either a Philips Brilliance40 or a General Electric LightSpeed scanner with collimations of 1.25, 2 and 2.5 millimeters and half overlapped or joint axial image reconstructions.

The segmentation results obtained on such a database were in good agreement with the analysis performed by an experienced radiologist on the native CT images. They demonstrate the robustness of the proposed methodology with respect to the anatomical variability and the liver opacifying degree. Fig. [6 illustrates some examples of 3D segmentation results for four patients at different perfusion phases. Note that such 3D segmentations must be interpreted with respect to the progression of the contrast bolus within the target vessels. When the degree of enhancement of a vessel decreases below some threshold, the vessel appears on the CT images as a collection of low-contrasted and disconnected small elements. Its components risk to be thus removed together with the overall noise during the multiresolution SMDC-CC based segmentation (Fig. 3(c)]. 


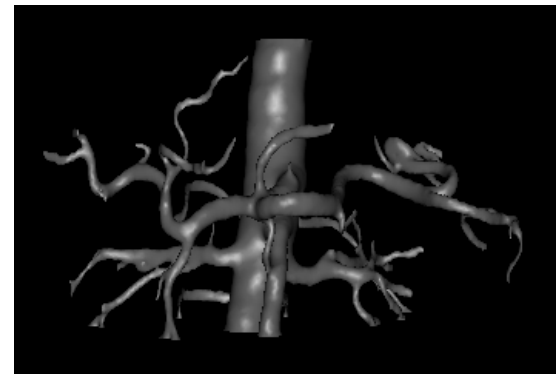

(a) Aorta and hepatic arteries at arterial phase.

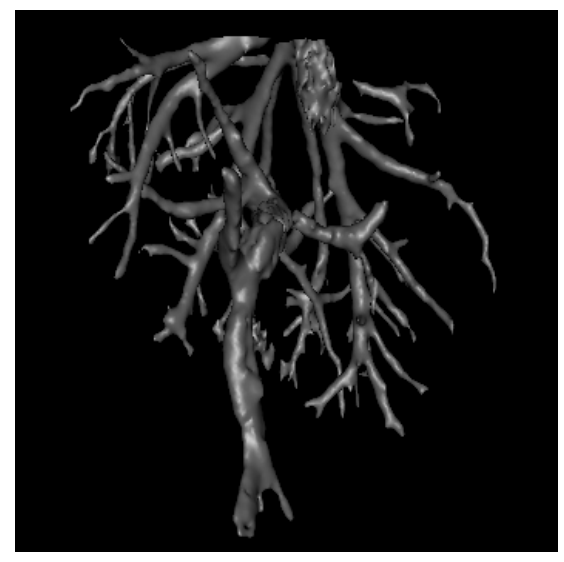

(c) Portal and hepatic veins at late portal phase.

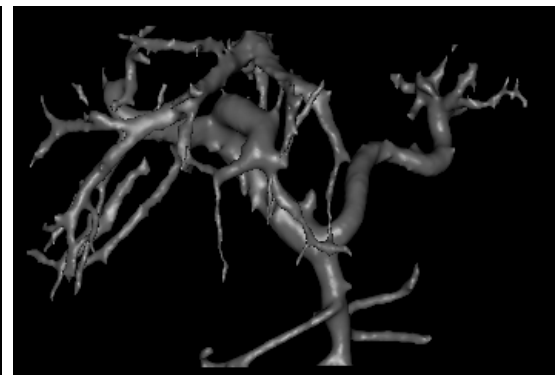

(b) Portal and hepatic veins at early portal phase.

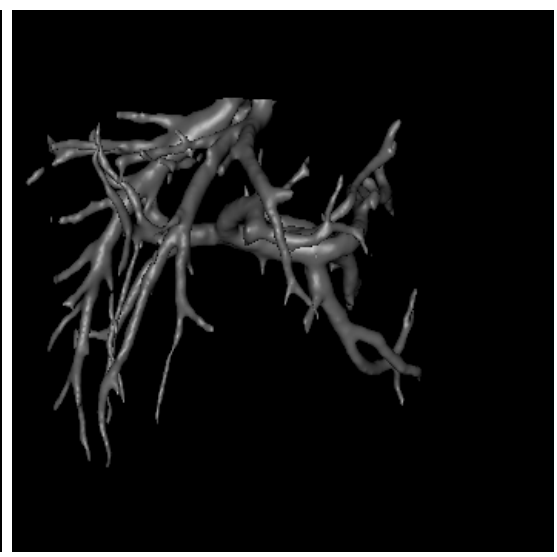

(d) Portal and hepatic veins at venous phase.

Fig. 6. Some results of $3 \mathrm{D}$ reconstructed hepatic vessels for different patients at different CT hepatic perfusion phases

In conclusion, the data acquisition triggering plays an important role in selecting the appropriate vessel enhancement. The analysis of portal and hepatic veins for preoperative purposes requires $\mathrm{CT}$ acquisitions at late portal phase which ensures the best contrast of such structures. Vessel topological analysis is possible after the $3 \mathrm{D}$ segmentation by means of a central axis-based description. In this respect, a central axis computation approach for tree-like structures and the related navigation/interaction tools have already been developed in the framework of pulmonary airways investigation. Their description is beyond the scope of this paper, but the interested reader could report to 13 .

\section{Conclusion}

This paper addressed the issue of 3D segmentation of liver vessels from 3D CT hepatic venography data acquired in clinical routine with various protocols. It 
developed an original and automated 3D reconstruction methodology based on topological and morphological operators combined in a multiresolution scheme. Such an approach made it possible to overcome the limitations raised by the CT image variability in terms of liver opacifying degree and noise presence. Tested at different perfusion phases, the developed segmentation methodology is currently under extensive validation in a clinical framework of liver surgery planning.

\section{References}

1. Y. Onodera et al., "Peripheral anatomic evaluation using 3D CT hepatic venography in donors: significance of peripheral venous visualization in living-donor transplantation", in American Journal of Radiology, 183, pp. 1065 -1070, 2004.

2. H.-O. Peitgen et al., "Risk analysis for liver surgery", Matematica et Cultura 2000, M. Emmer Ed., Springer, Milano, pp. 331-340, 2000.

3. D. Selle et al., "Analysis of vasculature for liver surgical planning", in IEEE Trans Med Imaging, 21(11), pp. 1344-1357, 2002.

4. B. Reitinger, A. Bornik, R. Beichel, G. Werkgartner, E. Sorantin, "Tools for Augmented Reality based Liver Resection Planning", SPIE Medical Imaging 2004, pp. 88-99, 2004.

5. H.-P. Meinzer, M. Thorn, C. Cardenas, "Computerized planning of liver surgery an overview", Computers \& Graphics 26, pp. 569-576, 2002.

6. H. Bourquain et al., "HepaVision2 - A software assistant for preoperative planning in living-related liver transplantation and oncologic liver surgery", Proc. CARS 2002, pp. 341-346, 2002.

7. P. Dokladal, C. Lohou, L. Perroton, G. Bertrand, "Liver blood vessel extraction by a 3D topological approach", Proc. MICCAI, 1679, pp. 98-104, 1999.

8. E. K. Fishman, B. S. Kuszyk, D. G. Heath, L Gao, B. Cabral, "Surgical planning for liver resection", IEEE Computer, pp. 64-72, 1996.

9. L. Soler et al., "Fully automatic anatomical, pathological, and functional segmentation from CT scans for hepatic surgery", Computer Aided Surgery, 6(3), pp. 131-142, 2001.

10. C. Fetita, F. Preteux, C. Beigelman-Aubry, P. Grenier, "Pulmonary airways: 3D reconstruction from multi-slice CT and clinical investigation", IEEE Transactions on Medical Imaging, 23(11), pp. 1353-1364, 2004.

11. L. Vincent, "Morphological gray scale reconstruction in image analysis: applications and efficient algorithms", IEEE Trans on Image Processing, 2(2), pp. 176-201, 1993.

12. F. Prêteux, "On a distance function approach for gray-level mathematical morphology", in Mathematical morphology in image processing, M. Dekker ed., E. R. Dougherty, 1992.

13. D. Perchet, C. Fetita, L. Vial, F. Preteux, G. Caillibotte, G. Sbirlea-Apiou, M. Thiriet, "Virtual investigation of pulmonary airways in volumetric computed tomography", Computer Animation \& Virtual Worlds, 15(3-4), pp. 361-376, 2004. 